\title{
A Difference Scheme for Solving Two Phase Stefan Problem of Heat Equation
}

\author{
(Errata to Vol. 16, No. 2, 1980, 313-341)
}

By

\author{
Tatsuo NoGI*
}

Professor S. Yotsutani pointed out mistakes of the paper titled above. The first point is about Lemma 4.1, whose proof was entrusted to Petrowsky's book [1]. But, the proof is valid only for the difference scheme with a uniform time step, and its method can not be applied for our scheme with variable time steps. Therefore, we must prove it with another method. In fact, we have proven it in Appendix A of our new paper (see Reference [2]).

The second point is about the algorithm in $\S 2$. It first has a trivial mistake in $4^{\circ}$. The suffix $n$ retards by 1 . It must be corrected as follows:

" $4^{\circ}$ if $J_{n+2}<J_{n+1}>J_{n}\left(J_{n+2}>J_{n+1}<J_{n}\right)$, then $J_{n+1}$ and $k_{n+1}$ are revised like $J_{n+1}=J_{n}$ and $k_{n+1}=b \sqrt{h} / \beta$, and return to the step $3^{\circ}$.

The last correction is, however, not sufficient because the obtained solution through such algorithm does not necessarily satisfy the Stefan condition. This also was pointed out by Professor S. Yotsutani.

Let us revise the algorithm and given some comments to show that the result of the paper itself does not get any change.

1 The algorithm on pp. 316-317 is replaced by the following :

$" 1{ }^{\circ}$

$$
u_{\jmath}^{0}=\phi_{\jmath}(1 \leqq j \leqq M-1), \quad y_{0}=J_{0} h=l .
$$

For $n=0,1,2, \cdots$, successively

$2.1^{\circ}$ if $\left(u_{J_{n}}^{n}\right)_{x}-\left(u_{J_{n}}^{n}\right)_{\bar{x}}>\beta h^{1 / 8}$, then $J_{n+1}=J_{n}+1$ and $k_{n+1}$ is determined from $(2.2)^{\prime}$,

$2.2^{\circ}$ if $\left(u_{J_{n}}^{n}\right)_{x}-\left(u_{J_{n}}^{n}\right)_{\bar{x}}<-\beta h^{1 / 8}$, then $J_{n+1}=J_{n}-1$ and $k_{n+1}$ is determined from $(2.2)^{\prime}$,

$2.3^{\circ}$ if $\mid\left(u_{J_{n}}^{n}\right)_{x}-\left(u_{J_{n}}^{n}\right)_{\bar{x}} \leqq \beta h^{1 / 8}$, then $J_{n+1}=J_{n}$ and $k_{n+1}=b h^{7 / 8} / \beta$,

Communicated by S. Hitotumatu, October 26, 1984.

* Department of Applied Mathematics and Physics, Faculty of Engineering, Kyoto University, Kyoto 606, Japan. 
$3^{\circ} \quad u^{n+1}$ is found from (2.1) and (2.4), and

$4^{\circ}$ if the obtained solution $u^{n+1}$ satisfies

$$
\left(u_{J_{n+1}}^{n+1}\right)_{x}-\left(u_{J_{n+1}^{n+1}}^{n+1}\right)_{\bar{x}}<-\beta h^{1 / 8} \quad\left(>\beta h^{1 / 8} \text {, alternatively }\right)
$$

in addition to

$$
\left(u_{J_{n}}^{n}\right)_{x}-\left(u_{J_{n}}^{n}\right)_{\bar{x}}>\beta h^{1 / 8}\left(<-\beta h^{1 / 8}\right)
$$

at the one step before, then $J_{n+1}$ are changed to be $J_{n}$, while $k_{n+1}$ are kept, and return again to the step $3^{\circ}$ and then skip $4^{\circ}$. (When $n=0, J_{-1}=J_{0}$ )"

By such change, we have

$$
\frac{b}{V} h<k_{n}<\frac{b}{\beta} h^{7 / 8} \quad(n=1,2,3, \cdots),
$$

instead of (2.7), where $V$ is an upper bound of $\left|\left(u_{J_{n}}^{n}\right)_{x}-\left(u_{J_{n}}^{n}\right)_{\bar{x}}\right|(n=1,2, \cdots)$.

2 Consider a step of number $n+1$ through $4^{\circ}$ of the above algorithm.

Lemma A. Suppose that, in addition to

$$
\left(u_{J_{n}}^{n}\right)_{x}-\left(u_{J_{n}}^{n}\right)_{\bar{x}}>\beta h^{1 / 8} \quad\left(\text { or }<-\beta h^{1 / 8}\right. \text {, alternatively), }
$$

the first trial of solution by $3^{\circ}$ produces the inequality

$$
v_{J_{n+1}}^{n+1}=\left(u_{J_{n+1}}^{n+1}\right)_{x}-\left(u_{J_{n+1}}^{n+1}\right)_{\bar{x}}<-\beta h^{1 / 8} \quad\left(>\beta h^{1 / 8}\right) 。
$$

Then, the value of $v_{J_{n+1}^{n+1}}^{n+1}$ by the second trial has the same sign, negative (positive), as that by the first trial, when $h$ is sufficiently small.

Its proof will given in the next section. We here use it to examine the Stefan condition. As in the paper, consider the continuous functions, $y_{\alpha}(t)$ and $v_{a_{ \pm}}(t)\left(h_{\alpha} \rightarrow 0\right.$ as $\alpha \rightarrow \infty)$ formed by interpolation of $\left\{y_{n}\right\}$ and $\left\{\left(u_{J_{n}}^{n}\right)_{x},\left(u_{J_{n}}^{n}\right)_{\bar{x}}\right\}$. Put $v_{\alpha}(t)=v_{\alpha+}(t)$ $-v_{\alpha-}(t)$, which also is an interpolated function from $\left\{v_{J_{n}}^{n}\right\}$. On such an interval $\left(t_{n}, t_{n+1}\right)$ that Lemma A holds, we can find a point $t_{n}^{\prime}$ such that

$$
v_{\alpha}\left(t_{n}^{\prime}\right)=0 \quad\left(t_{n}<t_{n}^{\prime}<t_{n+1}\right),
$$

since $v_{\alpha}\left(t_{n}\right)>0(<0)$ and $v_{\alpha}\left(t_{n+1}\right)<0(>0)$.

As in the paper (p. 325), we have

$$
\begin{aligned}
y_{\alpha}(t)= & y_{\alpha}(\tau)+\int_{\tau}^{t} \dot{y}(\theta) d \theta \\
= & y_{\alpha}(\tau)+\sum_{p=m+1}^{n-1} \operatorname{sign}_{p \leqq \theta<t_{p+1}}\left(\dot{y}_{\alpha}(\theta)\right) k_{p+1} \frac{h}{k_{p+1}} \\
& +\int_{\tau}^{t^{m+1}} \dot{y}_{\alpha}(\theta) d \theta+\int_{t_{n}}^{t} \dot{y}_{\alpha}(\theta) d \theta
\end{aligned}
$$


for any $\tau$ and $t\left(t_{m} \leqq \tau<t_{m+1}<t_{n} \leqq t<t_{n+1}\right)$. The formula (4.5) is still valid :

$$
y_{\alpha}(t)=y_{\alpha}(\tau)+\frac{1}{b} \sum_{p=m+1}^{n-1} k_{p+1} v_{\alpha}\left(t_{p}\right)+\int_{\tau}^{t_{m+1}} j(\theta) d \theta+\int_{t_{n}}^{t} \dot{y}_{a}(\theta) d \theta,
$$

where $\Sigma^{\prime}$ means summation except for number $p$ 's such that $\dot{y}\left(t_{p}+0\right)=0$, which occurrs when i) $\left|v_{\alpha}\left(t_{p}+0\right)\right|<\beta h^{1 / 8}$ holds or ii) the situation of Lemma A holds with $n=p$. We can add the corresponding terms of such excepted numbers to the right hand side of (3), only with error $O\left(h^{1 / 8}\right)$ :

$$
y_{\alpha}(t)=y_{\alpha}(\tau)+\frac{1}{b} \sum_{p=m+1}^{n-1} k_{p+1} v_{a}\left(t_{p}\right)+\frac{1}{b} \Sigma^{\prime \prime} k_{p+1} v\left(t_{p}\right)+\frac{1}{b} \Sigma^{\prime \prime \prime} k_{p+1} v_{\alpha}\left(t_{p}^{\prime}\right)
$$

$$
+\int_{\tau}^{t_{m+1}} \dot{y}_{\alpha}(\theta) d \theta+\int_{t_{n}}^{t} \dot{y}_{\alpha}(\theta) d \theta+O\left(h^{1 / 8}\right),
$$

since the terms corresponding to i) are such as

$$
\left|\Sigma^{\prime \prime} k_{p+1} v_{\alpha}\left(t_{p}\right)\right|<O\left(h^{1 / 8}\right) \Sigma^{\prime \prime} k_{p+1},
$$

and those corresponding to ii) are such as

due to (2).

$$
\Sigma^{\prime \prime \prime} k_{p+1} v_{\alpha}\left(t_{p}^{\prime}\right)=0
$$

Immediately from (4), we obtain the desired relation by taking $\alpha \rightarrow \infty$ :

$$
y(t)=y(\tau)+\frac{1}{b} \int_{\tau}^{t} v(\theta) d \theta
$$

This is the Stefan condition of the integral form. In the previous paper, we had missed out consideration about the terms corresponding to ii).

3 In order to prove Lemma $\mathrm{A}$, we will estimate the difference between two values of $\left(u_{J_{n+1}}^{n+1}\right)_{\bar{x}}$ by the first and second trials, and that between $\left(u_{J_{n+1}^{n+1}}^{n+1}\right)_{x}$ 's on such a time $t_{n+1}$ when the procedure $4^{\circ}$ is necessary. For distinction, put

$$
w_{\mp}^{n+1}=\left(u_{J_{n+1}}^{n+1}\right)_{\bar{x}} \text { or }\left(u_{J_{n+1}^{n+1}}^{n+1}\right)_{x} \text {, alternatively by the first trial }
$$

and

$$
z_{\mp}^{n+1}=\text { that by the second trial (in which } J_{n+1}=J_{n} \text { ). }
$$

Then, we have

Lemma B. $\left|w_{\mp}^{n+1}-z_{\mp}^{n+1}\right|<O\left(h^{3 / 8}\right)$.

From Lemma B, we have

$$
z_{+}^{n+1}-z_{-}^{n+1}=w_{+}^{n+1}-w_{-}^{n+1}+O\left(h^{3 / 8}\right) .
$$

By assumption, $\left|w_{+}^{n+1}-w_{-}^{n+1}\right|>\beta h^{1 / 8}$. Therefore, the sign of $z_{+}^{n+1}-z_{-}^{n+1}$ coinsides with that of $w_{+}^{n+1}-w_{-}^{n+1}$ for sufficiently small $h$. 
The remained problem is to prove Lemma B. We will here prove it only about $w_{-}^{n+1}-z_{-}^{n+1}$, since the other can be treated by the same way.

3.1 Consider to express $w_{-}^{n+1}$ and $z_{-}^{n+1}$ by using the Green function of the difference equation $c_{1}\left(u_{\jmath}^{n+1}\right)_{\bar{t}}=\left(u_{\jmath}^{n+1}\right)_{x \bar{x}}$ as done in [3]. Put

$$
G\left(x_{r}, \xi_{j} ; t_{n}, \tau_{p-1}\right)=\left\{\begin{array}{cc}
\frac{1}{2 \pi h} \int_{-\pi}^{\pi} \prod_{q=p}^{n} \Lambda_{q}^{-1}[\exp (-i(r-j) \omega)+\exp (-\imath(r+j-1) \omega)] d \omega & (\text { for } n \geqq p), \\
\frac{1}{h} \delta_{r, j}\left(\delta_{r, j}\right. \text { is Kronecker's delta) } & \text { (for } n=p-1),
\end{array}\right.
$$

where

$$
\Lambda_{q}=1+4 \lambda_{\exists} \sin ^{2} \frac{\omega}{2}, \quad \lambda_{q}=\frac{k_{r}}{c_{1} h^{2}}
$$

We then have

$$
\begin{aligned}
{[1+} & \left.c_{1}^{-1} k_{n+1} G_{\xi}\left(y_{n+1}^{\prime}, \eta_{n+1}^{\prime} ; t_{n+1}, \tau_{n}\right)\right] w_{-}^{n+1} \\
= & -\sum_{j=1}^{J} h G_{\xi}\left(y_{n+1}^{\prime}, \xi_{j} ; t_{n+1}, 0\right) \phi_{j}-c_{1}^{-1} \sum_{p=1}^{n} k_{p} G_{\xi}\left(y_{n+1}^{\prime}, \eta_{p} ; t_{n+1}, \tau_{p-1}\right) \tau_{-} \\
& \quad-c_{1}^{-1} \sum_{p=1}^{n+1} k_{p} G_{\xi \bar{\xi}}\left(y_{n+1}^{\prime}, \xi_{1} ; t_{n+1}, \tau_{p-1}\right) \psi_{1}^{p}
\end{aligned}
$$

and

$$
\begin{aligned}
{[1+} & \left.c_{1}^{-1} k_{n+1} G_{\xi}\left(y_{n+1}, \eta_{n+1} ; t_{n+1}, \tau_{n}\right)\right] z_{-}^{n+1} \\
= & -\sum_{j=1}^{J} h G_{\xi}\left(y_{n+1}, \xi_{j} ; t_{n+1}, 0\right) \phi_{j}-c_{1}^{-1} \sum_{p=1}^{n} k_{p} G_{\xi}\left(y_{n+1}, \eta_{p} ; t_{n+1}, \tau_{p-1}\right) v^{\underline{p}} \\
& \quad-c_{1}^{-1} \sum_{p=1}^{n+1} k_{p} G_{\xi \bar{\xi}}\left(y_{n+1}, \xi_{1} ; t_{n+1}, \tau_{p-1}\right) \psi_{1}^{p},
\end{aligned}
$$

where $v^{p}=\left(u_{J_{p}}^{p}\right)_{\bar{x}}, y_{n+1}^{\prime}=x_{J_{n+1}^{\prime}}, \eta_{n+1}^{\prime}=\eta_{J_{n+1}^{\prime}}$ and $J_{n+1}^{\prime}=J_{n} \pm 1($ means the first trial). For the second trial, $y_{n+1}=y_{n}$ and $\eta_{n+1}=\eta_{n}\left(J_{n+1}=J_{n}\right)$. Subtract (7) from (6). We then get

(8)

$$
\begin{aligned}
& {\left[1+c_{1}^{-1} k_{n+1} G_{\xi}\left(y_{n+1}^{\prime}, \eta_{n+1}^{\prime} ; t_{n+1}, \tau_{n}\right)\right]\left(w_{-}^{n+1}-z_{-}^{n+1}\right) } \\
&=--c_{1}^{-1} k_{n+1}\left[G_{\xi}\left(y_{n+1}^{\prime}, \eta_{n+1}^{\prime} ; t_{n+1}, \tau_{n}\right)-G_{\xi}\left(y_{n+1}, \eta_{n+1} ; t_{n+1}, \tau_{n}\right)\right] z_{-}^{n+1} \\
&-\sum_{j=1}^{J} h\left[G_{\xi}\left(y_{n+1}^{\prime}, \xi_{j} ; t_{n}, 0\right)-G_{\xi}\left(y_{n+1}, \xi_{j} ; t_{n+1}, 0\right)\right] \phi_{j} \\
&-c_{1}^{-1} \sum_{p=1}^{n} k_{p}\left[G_{\xi}\left(y_{n+1}^{\prime}, \eta_{p} ; t_{n+1}, \tau_{p-1}\right)-G_{\xi}\left(y_{n+1}, \eta_{p} ; t_{n+1}, \tau_{p-1}\right)\right] v^{p} \\
&-c_{1}^{-1} \sum_{p=1}^{n} k_{p}\left[G_{\xi \bar{\xi}}\left(y_{n+1}^{\prime}, \xi_{1} ; t_{n+1}, \tau_{p-1}\right)-G_{\xi \bar{\xi}}\left(y_{n+1}, \xi_{1} ; t_{n+1}, \tau_{p-1}\right)\right] \psi_{1}^{p} .
\end{aligned}
$$

In the following, we will estimate every term of (8) successively. 
3.2.

$$
1+c_{1}^{-1} G_{\xi}\left(y_{n+1}^{\prime}, \eta_{n+1}^{\prime} ; t_{n+1}, \tau_{n}\right)>\frac{1}{4}
$$

(See Lemma 2 of [3]).

3.3 We can write $G_{\xi}\left(y_{n+1}^{\prime}, \eta_{n+1}^{\prime} ; t_{n+1}, \tau_{n}\right)$ and $G_{\xi}\left(y_{n+1}, \eta_{n+1} ; t_{n+1}, \tau_{n}\right)$ as follows :

$$
\begin{aligned}
& G_{\xi}\left(y_{n+1}^{\prime}, \eta_{n+1}^{\prime} ; t_{n+1}, \tau_{n}\right) \\
& \quad=\frac{i}{\pi h^{2}} \int_{-\pi}^{\pi} \Lambda_{n+1}^{-1}\left[\exp \left(\frac{i \omega}{2}\right) \sin \frac{\omega}{2}-\exp \left(-i\left(2 J_{n+1}^{\prime}-\frac{1}{2}\right) \omega\right) \sin \frac{\omega}{2}\right] d \omega
\end{aligned}
$$

and

$$
\begin{aligned}
& G_{\xi}\left(y_{n+1}, \eta_{n+1} ; t_{n+1}, \tau_{n}\right) \\
& \quad=\frac{i}{\pi h^{2}} \int_{-\pi}^{\pi} \Lambda_{n+1}^{-1}\left[\exp \left(\frac{i \omega}{2}\right) \sin \frac{\omega}{2}-\exp \left(-i\left(2 J_{n}-\frac{1}{2}\right) \omega\right) \sin \frac{\omega}{2}\right] d \omega .
\end{aligned}
$$

Hence

$$
\begin{array}{r}
G_{\xi}\left(y_{n+1}^{\prime}, \eta_{n+1}^{\prime} ; t_{n+1}, \tau_{n}\right)-G_{\xi}\left(y_{n+1}, \eta_{n+1} ; t_{n+1}, \tau_{n}\right) \\
\quad=\left\{\begin{array}{r}
\left.-\frac{2}{\pi h^{2}} \int_{-\pi}^{\pi} \Lambda_{n+1}^{-1} \cos \left(2 J_{n}+1\right) \omega \sin ^{2} \frac{\omega}{2} d \omega \quad \text { (when } J_{n+1}^{\prime}=J_{n}+1\right) \\
\frac{2}{\pi h^{2}} \int_{-\pi}^{\pi} \Lambda_{n+1}^{-1}\left[\cos \left(2 J_{n}-1\right) \omega+\cos \left(2 J_{n}-2\right) \omega\right] \sin ^{2} \frac{\omega}{2} d \omega \\
\text { (when } \left.J_{n+1}^{\prime}=J_{n}-1\right) .
\end{array}\right.
\end{array}
$$

Consider the integrals here appeared. By integration by part, we have

$$
\begin{array}{r}
\int_{-\pi}^{\pi} \Lambda_{n+1}^{-1} \cos K \omega \sin ^{2} \frac{\omega}{2} d \omega=\frac{-1}{K} \int_{-\pi}^{\pi} \frac{\sin K \omega \sin (\omega / 2) \cos (\omega / 2)}{\left(1+4 \lambda_{n+1} \sin ^{2}(\omega / 2)\right)^{2} .} d \omega \\
\text { (K: an integer). }
\end{array}
$$

Since $|\sin K \omega|<|K \omega|<\pi\left|K \sin \frac{\omega}{2}\right|$ for $|\omega|<\pi$, we hence have

$$
\begin{aligned}
\left|\int_{-\pi}^{\pi} \Lambda_{n+1}^{-1} \cos K \omega \sin ^{2} \frac{\omega}{2} d \omega\right| & <\pi \int_{-\pi}^{\pi} \frac{\sin ^{2}(\omega / 2)}{\left(1+4 \lambda_{n+1} \sin ^{2}(\omega / 2)\right)^{2}} d \omega \\
& =\frac{\pi^{2}}{\left(1+4 \lambda_{n+1}\right)^{3 / 2}} .
\end{aligned}
$$

The last inequality itself is valid even for $K=0$. 'Therefore, we get

$$
\left.\left|\int_{-\pi}^{\pi} \Lambda_{n+1}^{-1} \cos K \omega \sin ^{2} \frac{\omega}{2} d \omega\right|<\frac{C h^{3}}{k_{n+1}^{3 / 2}} \quad \text { (C: a positive constant }\right)
$$

for any integer $K$. By applying this inequality on the right hand side of (10) and multiplying by $c_{1}^{-1} k_{n+1}$, we obtain

$$
\begin{gathered}
c_{1}^{-1} k_{n+1}\left|G_{\xi}\left(y_{n+1}^{\prime}, \eta_{n+1}^{\prime} ; t_{n+1}, \tau_{n}\right)-G_{\xi}\left(y_{n+1}, \eta_{n+1} ; t_{n+1}, \tau_{n}\right)\right| \\
\left.<\frac{C h}{\sqrt{k_{n+1}}}<C^{\prime} \sqrt{h} \quad \text { (due to }(1)\right) .
\end{gathered}
$$


Here and later, $C$ and $C^{\prime}$ are constants chosen appropriately each time.

\subsection{We next consider}

$$
-\sum_{j=1}^{J} h\left[G_{\xi}\left(y_{n+1}^{\prime}, \xi_{j} ; t_{n+1}, 0\right)-G_{\xi}\left(y_{n+1}, \xi_{j} ; t_{n+1}, 0\right)\right] \phi_{j}
$$

$$
\begin{aligned}
= & \sum_{j=1}^{J} h\left[G\left(y_{n+1}^{\prime}, \xi_{j} ; t_{n+1}, 0\right)-G\left(y_{n+1}, \xi_{j} ; t_{n+1}, 0\right)\right] \phi_{j \bar{x}} \\
& \left.+\left[G\left(y_{n+1}^{\prime}, \xi_{1} ; t_{n+1}, 0\right)-G\left(y_{n+1}, \xi_{1} ; t_{n+1}, 0\right)\right] \phi_{0} \text { (Note that } \phi_{J}=0\right) .
\end{aligned}
$$

Here,

$$
\begin{aligned}
& G\left(y_{n+1}^{\prime}, \xi_{j} ; t_{n+1}, 0\right)-G\left(y_{n+1}, \xi_{j} ; t_{n+1}, 0\right) \\
& \quad=\mp \frac{1}{\pi h} \int_{-\pi}^{\pi} \prod_{q=1}^{n+1} \Lambda_{q}^{-1}\left[\sin \left(J_{n}-j \pm \frac{1}{2}\right) \omega+\sin \left(J_{n}+j-1 \pm \frac{1}{2}\right) \omega\right] \sin \frac{\omega}{2} d \omega .
\end{aligned}
$$

By using Lemma 6 of [3], we get

$$
\begin{aligned}
& \left|G\left(y_{n+1}^{\prime}, \xi_{j} ; t_{n+1}, 0\right)-G\left(y_{n+1}, \xi_{j} ; t_{n+1}, 0\right)\right| \\
& <C h\left[\frac{1}{\left|J_{n}-j \pm(1 / 2)\right| h \sqrt{t_{n+1}-\hat{k}}}+\frac{\hat{k}}{\left|J_{n}-j \pm(1 / 2)\right| h\left(t_{n+1}-\hat{k}\right)^{3 / 2}}\right. \\
& \left.\quad+\frac{1}{\left|J_{n}+j-1 \pm(1 / 2)\right| h \sqrt{t_{n+1}-\hat{k}}}+\frac{\hat{k}}{\left|J_{n}+j-1 \pm(1 / 2)\right| h\left(t_{n+1}-\hat{k}\right)^{3 / 2}}\right]
\end{aligned}
$$

where $\hat{k}=\max k_{p}(1 \leqq p \leqq n+1)$. Hence

$$
\begin{aligned}
& \left|\sum_{j=1}^{J} h\left[G\left(y_{n+1}^{\prime}, \xi_{j} ; t_{n+1}, 0\right)-G\left(y_{n+1}, \xi_{j} ; t_{n+1}, 0\right)\right] \phi_{j \bar{x}}\right| \\
& <C h|\ln h|\left(\frac{1}{\sqrt{t_{n+1}-\hat{k}}}+\frac{\hat{k}}{\left(t_{n+1}-\hat{k}\right)^{3 / 2}}\right) .
\end{aligned}
$$

For a finite $t=t_{n+1}$, we have $t_{n+1}>\frac{2 b}{\beta} h^{7 / 8}$ for sufficiently small $h$. Then, $t_{n+1}-\hat{k}>\frac{1}{2} t_{n+1}>\frac{b}{\beta} h^{7 / 8}$ (due to (1)). Thus

$$
\left|\sum_{j=1}^{J} h\left[G\left(y_{n+1}^{\prime}, \xi_{j} ; t_{n+1}, 0\right)-G\left(y_{n+1}, \xi_{j} ; t_{n+1}, 0\right)\right] \phi_{j \bar{x}}\right|<C h^{9 / 16}|\ln h| .
$$

Also by (13), we have

$$
\left|G\left(y_{n+1}^{\prime}, \xi_{1} ; t_{n+1}, 0\right)-G\left(y_{n+1}, \xi_{1} ; t_{n+1}, 0\right)\right|\left|\phi_{0}\right|<C h^{9 / 16} .
$$

By applying (14) and (15) on (12), we finally obtain

(16) $\left|-\sum_{j=1}^{J} h\left[G_{\xi}\left(y_{n+1}^{\prime}, \xi_{j} ; t_{n+1}, 0\right)-G_{\xi}\left(y_{n+1}, \xi_{j} ; t_{n+1}, 0\right)\right] \phi_{j}\right|<C h^{9 / 16}|\ln h|$.

3.5 It is essential for our purpose to estimate the sum

$$
-c_{1}^{-1} \sum_{p=1}^{n} k_{p}\left[G_{\xi}\left(y_{n+1}^{\prime}, \eta_{p} ; t_{n+1}, \tau_{p-1}\right)-G_{\xi}\left(y_{n+1}, \eta_{p} ; t_{n+1}, \tau_{p-1}\right)\right] v^{p} .
$$


Here

$$
\begin{aligned}
& G_{\xi}\left(y_{n+1}^{\prime}, \eta_{p} ; t_{n+1}, \tau_{p-1}\right) \\
& =\frac{i}{\pi h^{2}} \int_{-\pi}^{\pi} \sum_{q=p}^{n+1} \Lambda_{q}^{-1}\left[\exp \left(-i\left(J_{n+1}^{\prime}-J_{p}-\frac{1}{2}\right) \omega\right)\right. \\
& \left.\quad-\exp \left(-i\left(J_{n+1}^{\prime}+J_{p}-\frac{1}{2}\right) \omega\right)\right] \sin \frac{\omega}{2} d \omega
\end{aligned}
$$

and

$$
\begin{aligned}
G_{\xi}\left(y_{n+1}, \eta_{p} ; t_{n+1}, \tau_{p-1}\right) & \\
= & \frac{i}{\pi h^{2}} \int_{-\pi}^{\pi} \prod_{q=p}^{n+1} \Lambda_{q}^{-1}\left[\exp \left(-i\left(J_{n}-J_{p}-\frac{1}{2}\right) \omega\right)\right. \\
& \left.-\exp \left(-i\left(J_{n}+J_{p}-\frac{1}{2}\right) \omega\right)\right] \sin \frac{\omega}{2} d \omega .
\end{aligned}
$$

Hence

$$
\begin{aligned}
& G_{\xi}\left(y_{n+1}^{\prime}, \eta_{p} ; t_{n+1}, \tau_{p-1}\right)-G_{\xi}\left(y_{n+1}, \eta_{p} ; t_{n+1}, \tau_{p-1}\right) \\
& \quad= \pm \frac{2}{h^{2}} \int_{-\pi}^{\pi} \prod_{q=p}^{n+1} \Lambda_{q}^{-1}\left[\cos \left(J_{n}-J_{p}-\frac{1 \mp 1}{2}\right) \omega-\cos \left(J_{n}+J_{p}-\frac{1 \mp 1}{2}\right) \omega\right] \sin ^{2} \frac{\omega}{2} d \omega
\end{aligned}
$$

and further

$$
\begin{aligned}
& \left|G_{\xi}\left(y_{n+1}^{\prime \prime}, \eta_{p} ; t_{n+1}, \tau_{p-1}\right)-G_{\xi}\left(y_{n+1}, \eta_{p} ; t_{n+1}, \tau_{p-1}\right)\right| \\
& \quad<\frac{4}{\pi h^{2}} \int_{-\pi}^{\pi} \prod_{q=p}^{n+1} \Lambda_{q}^{-1} \sin ^{2} \frac{\omega}{2} .
\end{aligned}
$$

We can estimate the right hand side by using Lemma 5 of [3]:

$$
\frac{4}{\pi h^{2}} \int_{-\pi}^{\pi} \prod_{q=p}^{n+1} \Lambda_{q}^{-1} \sin ^{2} \frac{\omega}{2} d \omega=\left\{\begin{array}{l}
\frac{2 c_{1}^{3 / 2} h}{\sqrt{2\left(t_{n+1}-\tau_{p-1}-\hat{k}\right)^{3 / 2}}} \text { or } \\
\frac{c_{1}^{3 / 2} h}{\sqrt{k_{n} k_{n+1}}\left(\sqrt{k_{n}}+\sqrt{\left.k_{n+1}\right)}\right.}
\end{array},\right.
$$

where $\hat{k}=\hat{k}(p, n+1)=\max _{q=p, p+1, \cdots, n+1} k_{q}$. For estimation of the sum (17) with (18) and (19), we divide the sum into two parts: i) for $t_{n+1}-\tau_{p-1} \geqq \frac{2 b}{\beta} h^{7 / 8}$ and ii) for $t_{n+1}-\tau_{p-1}<\frac{2 b}{\beta} h^{7 / 8}$. In the former part, we use the upper half of (19) and notice that $t_{n+1}-\tau_{p-1}-\hat{k}>\frac{1}{2}\left(t_{n+1}-\tau_{p-1}\right)$ by (1). Hence

$$
\frac{4}{\pi h^{2}} \int_{-\pi}^{\pi} \prod_{q=p}^{n+1} \Lambda_{q}^{-1} \sin ^{2} \frac{\omega}{2} d \omega<\frac{C h}{\left(t_{n+1}-\tau_{p-1}\right)^{3 / 2}} \text { for } \quad t_{n+1}-\tau_{p-1}>\frac{2 b}{\beta} h^{7 / 8}
$$

Again by (1), we also have

$$
\frac{4}{\pi h^{2}} \int_{-\pi}^{\pi} \prod_{q=p}^{n+1} \Lambda_{q}^{-1} \sin ^{2} \frac{\omega}{2} d \omega<\frac{C}{\sqrt{h}} .
$$


from the lower half of (19). Thus, the concerned sum (17) is estimated as follows :

(22)

$$
\begin{gathered}
\left|-c_{1}^{-1} \sum_{p=1}^{n} k_{p}\left[G_{\xi}\left(y_{n+1}^{\prime}, \eta_{p} ; t_{n+1}, \tau_{p-1}\right)-G_{\xi}\left(y_{n+1}, \eta_{p} ; t_{n+1}, \tau_{p-1}\right)\right] v^{\underline{p}}\right| \\
<C\left[h \cdot \int_{t_{n+1}-\tau>(2 b / \beta) h^{7 / 8}} \frac{d \tau}{\left(t_{n+1}-\tau\right)^{3 / 2}}+h^{7 / 8} \cdot \frac{1}{\sqrt{h}}\right]<C h^{3 / 8} .
\end{gathered}
$$

3.6 Finally, we consider

$$
\begin{aligned}
& -c_{1}^{-1} \sum_{p=1}^{n+1} k_{p}\left[G_{\xi \bar{\xi}}\left(y_{n+1}^{\prime}, \xi_{1} ; t_{n+1}, \tau_{p-1}\right)-G_{\xi \bar{\xi}}\left(y_{n+1}, \xi_{1} ; t_{n+1}, \tau_{p-1}\right)\right] \psi_{1}^{p} \\
& =\sum_{p=1}^{n+1} k_{p}\left[G_{\tau}\left(y_{n+1}^{\prime}, \xi_{1} ; t_{n+1}, \tau_{p-1}\right)-G_{\tau}\left(y_{n+1}, \xi_{1} ; t_{n+1}, \tau_{p-1}\right)\right] \psi_{1}^{p} \\
& =-\sum_{p=1}^{n+1} k_{p}\left[G\left(y_{n+1}^{\prime}, \xi_{1} ; t_{n+1}, \tau_{p-1}\right)-G\left(y_{n+1}, \xi_{1} ; t_{n+1}, \tau_{p-1}\right)\right] \psi_{1 \bar{\tau}}^{p} \\
& \quad-\left[G\left(y_{n+1}^{\prime}, \xi_{1} ; t_{n+1}, 0\right)-G\left(y_{n+1}, \xi_{1} ; t_{n+1}, 0\right)\right] \psi_{1}^{0} .
\end{aligned}
$$

Here

$$
\begin{aligned}
& G\left(y_{n+1}^{\prime}, \xi_{1} ; t_{n+1}, \tau_{p-1}\right)-G\left(y_{n+1}, \xi_{1} ; t_{n+1}, \tau_{p-1}\right) \\
& \quad= \pm \frac{1}{\pi h} \int_{-\pi}^{\pi} \prod_{q=p}^{n+1} \Lambda_{q}^{-1}\left[\sin \left(J_{n}-1 \pm \frac{1}{2}\right) \omega+\sin \left(J_{n} \pm \frac{1}{2}\right) \omega\right] \sin \frac{\omega}{2} d \omega .
\end{aligned}
$$

For estimation of the last integral, we apply Lemma 6 of [3]. By taking the same way of consideration for $t_{n+1}-\tau_{p-1}-\hat{k}$ as in 3.5 , we get

$$
\begin{aligned}
& \left|G\left(y_{n+1}^{\prime}, \xi_{1} ; t_{n+1}, \tau_{p-1}\right)-G\left(y_{n+1}, \xi_{1} ; t_{n+1}, \tau_{p-1}\right)\right| \\
& \quad<C h\left[\frac{1}{\left(J_{n}-1 \pm(1 / 2)\right) h \sqrt{t_{n+1}-\tau_{p-1}}}+\frac{h^{7 / 8}}{\left(J_{n}-1 \pm(1 / 2)\right) h\left(t_{n+1}-\tau_{p-1}\right)^{3 / 2}}\right. \\
& \left.\quad+\frac{1}{\left(J_{n} \pm(1 / 2)\right) h \sqrt{t_{n+1}-\tau_{p-1}}}+\frac{h^{7 / 8}}{\left(J_{n} \pm(1 / 2)\right) h\left(t_{n+1}-\tau_{p-1}\right)^{3 / 2}}\right]
\end{aligned}
$$

for $t_{n+1}-\tau_{p-1}>\frac{2 b}{\beta} h^{7 / 8}$, or

Hence

$$
<C\left[\frac{\sqrt{t_{n+1}-\tau_{p-1}}}{\left(J_{n}-1 \pm(1 / 2)\right) h}+\frac{\sqrt{t_{n+1}-\tau_{p-1}}}{\left(J_{n} \pm(1 / 2)\right) h}\right] .
$$

$$
\begin{aligned}
& \left|-\sum_{p=1}^{n+1} k_{p}\left[G\left(y_{n+1}^{\prime}, \xi_{1} ; t_{n+1}, \tau_{p-1}\right)-G\left(y_{n+1}, \xi_{1} ; t_{n+1}, \tau_{p-1}\right)\right] \psi_{1 \bar{\tau}}^{p}\right| \\
& \quad<C h\left[\int_{t_{n+1}-\tau \geqq(2 b / \beta) h 7 / 8} \frac{d \tau}{\sqrt{t_{n+1}-\tau}}+h^{7 / 8} \int_{t_{n+1}-\tau \geqq(2 b / \beta) h 7 / 8} \frac{d \tau}{\left(t_{n+1}-\tau\right)^{3 / 2}}\right] \\
& \quad+C \int_{t_{n+1} \tau<(2 b / \beta) h 7 / 8} \sqrt{t_{n+1}-\tau} d \tau \\
& \quad<C h^{21 / 16}
\end{aligned}
$$


and

$$
\begin{aligned}
& \left|\left[G\left(y_{n+1}^{\prime}, \xi_{1} ; t_{n+1}, 0\right)-G\left(y_{n+1}, \xi_{1} ; t_{n+1}, 0\right)\right] \phi_{1}^{0}\right| \\
& \quad<C h\left[\frac{1}{\sqrt{t_{n+1}}}+\frac{h^{7 / 8}}{t_{n+1}^{3 / 2}}\right]<C h^{9 / 16} \text { for } t_{n+1}>\frac{2 b}{\beta} h^{7 / 8} .
\end{aligned}
$$

We here used the tacit assumption that $y_{n}=J_{n} h$ is far from the boundary $x=0$ by a finite distance, and so is $t_{n+1}$ from $t=0$. By applying (24) and (25) on (23), we get

$$
\left|-c_{1}^{-1} \sum_{p=1}^{n+1} k_{p}\left[G_{\xi \bar{\xi}}\left(y_{n+1}^{\prime}, \xi_{1} ; t_{n+1}, \tau_{p-1}\right)-G_{\xi \bar{\xi}}\left(y_{n+1}, \xi_{1} ; t_{n+1}, \tau_{p-1}\right)\right] \psi_{1}^{p}\right|<C h^{9 / 16} 。
$$

3.7 By applying (9), (11), (16), (22) and (26) upon (8), we obtain, in conclusion, the desired estimate of Lemma B.

\section{Acknowledgements}

The author thanks deeply to Professor S. Yotsutani for indication of some errors in the original paper and useful discussion.

\section{References}

[1] Petrowsky, I.G., Partial Differential Equations, London Iliffe Books Ltd. 1967. (Originally published in Moscow in 1961 by Fizmatgiz under the title Lektsii ob uravneniyakh s chastnymi proizvodnymi).

[2] Nogi, T., A Mathematical One-Dimensional Model of Supercooling Solidification, Publ. RIMS, Kyoto Univ., to appear.

[3] Nogi, T., A Differences scheme for solving the Stefan problem, Publ. RIMS, Kyoto Univ., 9 (1974), 543-575. 
